\title{
Vibration Protection of Mechanical Systems Consisting of Solid and Deformable Bodies
}

\author{
Ismail I. Safarov, and Teshaev M. Khudoyberdiyevich
}

\begin{abstract}
In this paper active vibration protection of mechanical systems consisting of solid and deformable bodies is considered. To actively control the oscillations of dissipative mechanical systems, a constructive method is used to determine the structure of the reaction forces of servo constraints. As an example, we consider the system with a finite number of degrees of freedom. Numerical results for various harmonic are also given.
\end{abstract}

Index Terms-Active Vibration Protection; Mechanical System; Oscillations; Dissipation; Harmonic Load.

\section{INTRODUCTION}

The increase in the productivity and efficiency of machines, the increase in the speed of motion of working organs, the reduction of the material consumption, the increased loads due to vibrations and shocks, the need to ensure reliable operation of equipment and safe working conditions are factors that determine attention to the tasks of vibration protection. In this respect, structural research methods that are based on the idea of using a special class of mathematical models are rather promising. At the same time, there are a number of difficulties connected with the possibilities of constructing adequate mathematical models, evaluating the role and dissipative properties of system elements, choosing methods for describing the motion and determining the possibilities for changing the dynamic state of objects in the search for and choosing certain constructive and technical solutions.

\section{About Some Methodological Bases of APPLICATION OF GEOMETRIC SERVO CONSTRAINTS TO ACTIVE VIBRATION PROTECTION SYSTEM}

Inadequate vibration stability of the technology in many cases does not allow to use it effectively due to the occurrence of increased fluctuations, as a result of which restrictions on the performance parameters of machines are reduced, which reduce their technical characteristics and competitiveness.

An effective method of increasing the vibration stability of machines and structures, as well as improving the quality of vibration isolation devices, equipment and workplaces is the active damping of oscillations based on the use of systems for automatic control of the oscillatory process [1][3].

Published on September 20, 2018.

I.I. Safarov is located with the Department of Mathematics, the Faculty of Economics, the Tashkent Chemical Technology Institute, the Republic of Uzbekistan. (E-mail: safarov54@mail.ru).

T. M. Khudoyberdiyevich is with Bukhara Engineering-Technological Institute, Bukhara, Republic of Uzbekistan.
On active vibration protection systems and its application. The modern dynamics of machines is an interdisciplinary scientific and technical space in which the problems of the theory of oscillations, theoretical and applied mechanics, the theory of mechanisms and machines, machine science, mechatronics, robotics, vibration diagnosis and vibration mechanics intersect. Sectoral coloring introduces essential elements of specificity, often requiring special approaches based on taking into account nonlinear effects, peculiarities of interaction of working organs with the external environment and differences in systems of external influences and disturbances. Famous systems of normative documents consolidate the experience gained in the field of theory and practice and allow in many cases to use, seek and find rational solutions. Recently, the direction connected with the problems of the dynamics of controlled mechanical systems, implementing ideas in the theory of oscillations and its numerous applications, connected with the introduction of feedbacks, has developed significantly. Studies in the field of protecting various objects from vibrations and shocks have received intensive development among scientists of our country and abroad [4]-[6]. Problems of the dynamics of control systems are considered in [7]-[9]. In our country, the idea of using an inverse negative connection is well perceived at the physical level when considering oscillatory motion in mechanical systems. Such problems were posed in [10], [11]. Self-organization of the steady-state relative motion caused by disturbances, and the response of the system to various kinds of influences are associated with various forms of introduction of feedbacks. In these papers, theoretical aspects of the problem of introducing additional connections into mechanical oscillatory systems and their implementation on the basis of the use of special technical means that consume the energy of additional sources.

The development of ideas for the introduction of feedbacks, if we consider the first stages in the formation of concepts associated with the use of feedback in the dynamics of mechanical oscillatory systems, relies on the use of structural methods of investigation.

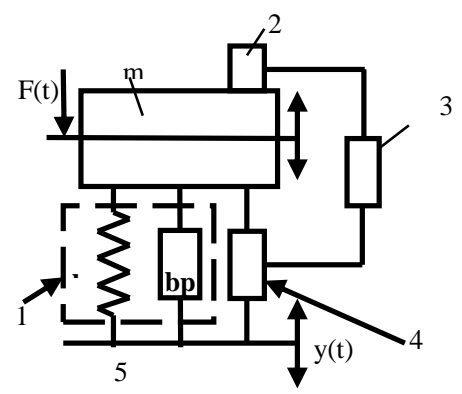

Fig. 1. The design scheme of vibration protection systems 
1-Passive part of ejection systems. 2- The sensor. 3- The device for signal conversion. 4- Active part (servo). 5Foundation.

The theory of automatic control, which developed with considerable advance over mechanics, predetermined the choice of the mathematical apparatus and the direction of research, which became one of the sections of the modern theory of oscillations [12], [13].

The subsequent presentation is based on the notion that any mechanical oscillatory system can be compared with a dynamically equivalent structural diagram of an automatic control system. All kinds of connections - direct, inverse have a physical interpretation; external influences (power and kinematic) take the form of the corresponding input signals, and the parameters of the state of the protection object become output signals.

The objects used in engineering and their dynamics are quite complex technical systems. Their various dynamic properties are reflected by computational schemes of various complexity and corresponding mathematical models. The above schemes are used to isolate the protection object, the system of external influences and the main constituent elements, that is, all that is necessary for compiling mathematical models of ejection protection systems. Mathematical models in the form of a system of differential equations are considered in [14],[15].

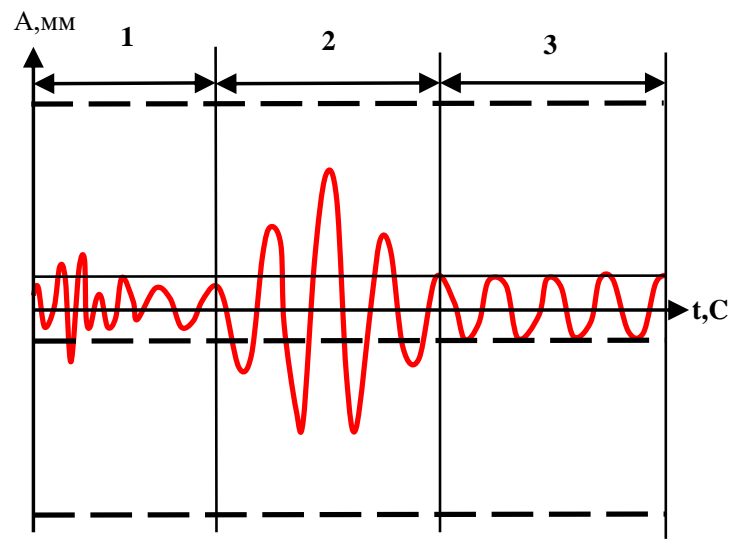

Fig. 2. Change the motion of the REA object as a function of time. 1-Changing the frequency of external vibration. 2- Entering into resonance. 3- The steady state

Equations of motion are formed on a formalized basis, using the d'Alembert principle, or Lagrange equations of the second kind [16]. Since the objects are complex, the set of motions in question is quite extensive, therefore, one can speak of the existence of a certain class of models reflecting different properties of vibrational motions. However, in the above-mentioned papers, the questions of composing a closed system of equations were not considered. Therefore, in this paper an attempt is made to compile closed equations of motion, taking into account the controllability of the system.Practically in any branch of industrial activity, problems arise in assessing the level of the dynamic state of machines and equipment, that is, assessing their level of vibration, searching for and developing state management tools. The figure shows the graph of the change in different modes of oscillation (Fig. 2). From Fig. 2. It can be seen that the considered region of the object's oscillations is divided into three parts:
- change of the object's displacements under the influence of the vibration action (before the resonance case);

- inclusion into resonance;

- stored area.

Equation of vibration and reaction force. If we use the Boltzmann-Voltaire relations to describe the relationship between stresses and strains, then

$$
[a]\left\{q^{\prime \prime}\right\}+[c]\{q\}=\{Q(t)\}
$$

where

$$
\widehat{C}_{i j} \phi(t)=C_{i j}\left[\phi(t)-\int_{0}^{t} R_{i j}(t-\tau) \phi(\tau) d \tau\right]
$$

Together with the assumption of the vibrational character of the motion, this will allow us to apply the freezing procedure [16], [17], which leads to the following complex physical relationships for deformable elements of zero volume [16], [17]:

$F_{e}=-c_{e} \Delta e=-c_{e}\left[1-\Gamma_{e}^{c}\left(\omega_{R}\right)-i \Gamma_{e}^{s}\left(\omega_{R}\right)\right] \Delta e$

For elements with distributed stiffness this relation will have the form:

$\sigma_{i j}=\bar{\lambda}_{n} \varepsilon_{n i} \delta_{j i}+2 \bar{\mu}_{n} \varepsilon_{n i}, \quad S=S_{1}+S_{2} \quad, \quad n=1,2,3, \ldots, S$,

where

$$
\begin{aligned}
& \bar{\lambda}_{n}=\lambda_{n}\left[1-\Gamma_{n \lambda}^{c}\left(\omega_{R}\right)-i \Gamma_{n \mu}^{s}\left(\omega_{R}\right)\right\rfloor \\
& \Gamma_{\lambda, m}^{c}(\omega)=\int_{0}^{\infty} R_{\lambda, m}(\tau) \cdot \cos \omega \tau d \tau ; \quad \Gamma_{\lambda, \mu}^{s}(\omega)=\int_{0}^{\infty} R_{\lambda, \mu}(\tau) \sin \omega \tau d \tau
\end{aligned}
$$

$F_{e}$ - force in the I - th concentrated element, $\Delta e$-the elongation of this element; $\bar{C}_{e}, \sigma_{i j}, \varepsilon_{i j}$-its complex rigidity, stress and deformation in an element of zero volume; $\Gamma_{e}^{c}, \Gamma_{e}^{s}, \Gamma_{n \lambda}^{c}, \Gamma_{n \lambda}^{s}, \Gamma_{n \mu}^{c}, \Gamma_{n \mu}^{s}$ - the sine and cosine of the Fourier image of the relaxation kernels of the i-th concentrated element and the $n$-the distributed element; $\omega_{R}$ the real part of the complex oscillation frequency of the system. In this case, the frequency will be complex for Eigen values, real-for forced oscillations. In the first case, the complex Eigen frequency is the frequency of damped oscillations, the imaginary part is the coefficient of damping of the natural oscillations of the system. In the second case, $w_{R}$ coincides with the frequency of forced oscillations. For natural vibrations, the relations (2) are approximate, and for forced oscillations, they are exact.

If in the problems of vibration protection and vibration isolation or control of the dynamic state of an object, active elements associated with the attraction of additional (external) energy sources are introduced, the mathematical models can be represented by matrix-vector differential equations of the first order in the normal form:

$$
\{\dot{x}\}=[A]\{x\}+\{F(t)\}
$$


Where $\{x\}-n$-dimensional state vector (phase coordinates) of the model; $[A]$-real or complex $(n \times n)$ matrix of arbitrary structure; $\{F(t)\}-n$-dimensional vector function of external influences. In those cases when the system is more complex, that is, the system is not a chain system, the matrix $[B]$ and $[C]$ can become absolutely "dense".As an example, consider oscillations of a system with two degrees of freedom (Fig. 4). Then the system of differential equations (1) - (3) takes the form

$$
\begin{gathered}
{[M]\{X\}+\left[B_{1}\right]\left\{\dot{X}_{1}\right\}+\left[B_{2}\right]\left\{\dot{X}_{2}\right\}+\left[C_{1}\right]\left\{X_{1}\right\}+\left[C_{2}\right]\left\{X_{2}\right\}=\{F(t)\}+\{\eta(t)\} ;} \\
L \frac{d i}{d t}+R i+B e\left(\dot{x}_{3}-\dot{x}_{2}\right)=\left(u_{b x}-u_{d p}\right) K_{1} ; \\
u_{d p}=K_{d p}\left(x_{3}-x_{1}\right) ; u_{b x}=K_{0} K_{d p} x_{1},
\end{gathered}
$$

where

$$
\begin{aligned}
& {[M]=\left(\begin{array}{ccc}
m_{1} & 0 & 0 \\
0 & m_{2} & 0 \\
0 & 0 & m_{3}
\end{array}\right),[B]=\left(\begin{array}{ccc}
b_{1} & 0 & 0 \\
0 & b_{1} & 0 \\
0 & 0 & b_{3}
\end{array}\right),\left[B_{1}\right]=\left(\begin{array}{ccc}
0 & 0 & 0 \\
0 & b_{2} & 0 \\
0 & 0 & 0
\end{array}\right),\{X\}=\left\{\begin{array}{l}
x_{1} \\
x_{2} \\
x_{3}
\end{array}\right\}} \\
& \left\{\dot{X}_{1}\right\}=\left\{\begin{array}{l}
\dot{x}_{1}-\dot{x}_{2} \\
\dot{x}_{2}-\dot{x}_{1} \\
\dot{x}_{3}-\dot{x}_{2}
\end{array}\right\},\left\{\dot{X}_{2}\right\}=\left\{\begin{array}{c}
0 \\
x_{2} \\
0
\end{array}\right\},\left[C_{1}\right]=\left(\begin{array}{ccc}
c_{1} & 0 & 0 \\
0 & c_{2} & 0 \\
0 & 0 & c_{3}
\end{array}\right),\left[C_{2}\right]=\left(\begin{array}{ccc}
0 & 0 & 0 \\
0 & c_{2} & 0 \\
0 & 0 & 0
\end{array}\right)
\end{aligned}
$$

$u$ - control voltage on the coil winding; i - current strength; $B \ell i$-electrodynamics force; L, R - inductance and active resistance of the coil; $\mathrm{B}$ is the magnetic induction; $\ell$ is the total length of the conductor; $K_{d p}$ - displacement sensor transfer coefficient; $K_{0}-$ amplification factor; $K_{l}-$ gain in the servo system.

Structural methods of research based on geometric servo constraints represent an original direction based on the use of mathematical models in the form of structural schemes and mechanical systems built using multicolor elements, which stimulated the development of methods for automated research of mechanical systems and methods of analog electromechanical modeling. The development of vibration protection and vibration isolation technologies for machines and equipment is on the way to turning the protection systems, half-beings, into an automatic control system, and the general trend that reflects the essence of the process is that the feedback in the protection systems involves not only the primary elements (springs, dampers and others), but also sensors of the device for information processing, amplifiers, corrective circuits, regulators and actuators in the form of servo drives.

We note that the general tendency and complication of the control systems themselves, which in their most developed forms are realized as neural networks, use the effects of adaptation and self-organization of the movement. At the same time, the very fact of integration of technical means, realizing complex forms of introduction of feedbacks, is quite obvious. In addition to conventional springs and dampers, vibration structures, motion transformation mechanisms, mechanical circuits, servo drives are introduced into consideration.

Dynamic servo constraint is introduced by us as an additional negative feedback.

An important circumstance is that static reactions at the required points of the mechanical system can be determined on the basis of the use of structural circuits of dynamically equivalent automatic control systems. Note that the use of structural approaches is associated with certain conventions, since structural schemes, in the usual sense, are used for periodic signals. We also note that when considering static problems and static reactions caused by weight forces or other constant forces formed by special devices, the transfer functions used are Laplace image relations of output signals as displacements with input signals in the form of constant forces. The proposed method can be extended to systems in which the masses of inertial elements can be represented by solids.

Generalized dynamic servo constraint can be formed on the basis of an extended set of typical elements by combining the types of elements of different types in the structure according to the rules of parallel and sequential addition. The generalized connection can be realized by mechanisms that produce various generalized forces, such as, for example, mechanical, electromechanical, hydraulic, electro hydraulic, etc. The methodology for determining the reactions of out gassing systems to external influences relies on frequency methods of the theory of automatic control and makes it possible to obtain the transfer functions of outtake protection systems taking into account the physical features of the implementation of generalized dynamic relationships. As already noted, they can be diverse in physical nature, differently included in the objectives:

- Mechanical oscillatory systems, which are the design schemes of various vibration protection and vibration isolation systems for machines, equipment and aggregates, can be represented by structural schemes of dynamically equivalent automatic control systems;

- Direct and inverse connections on structural analogues correspond to physically realizable constraints in the form of known elastic and damping devices, on the whole, forming a completely defined set of typical elements of mechanical systems based on the theory of geometric servo connections;

- Developed the algorithm and methodological basis for constructing mechanical systems based on the use of an extended set of typical elements and certain rules for their connection;

- The possibilities of constructing a generalized approach in the dynamic synthesis of mechanical systems are demonstrated using the techniques of simplifying the transfer function of the additional constraint, which allows obtaining the available results in problems of vibration protection and vibration isolation, as particular cases of a general approach;

- The proposed approach allows not only to unify the results of predecessors obtained in numerous studies on a unified basis, but also to develop a methodological basis and scientifically grounded methods of searching for and developing new technical means for changing and managing the dynamic state in problems of protection from vibrations and shocks.

Summarizing the results of the research, I would like to emphasize that structural approaches are a rational tool for selecting, researching and designing active emission protection systems. This involves a set of issues related to 
the evaluation of the dynamic properties of active outtake protection systems for various types of generalized dynamic relationships [17]-[19].

The formulation of the theorem. If the linear oscillatory dissipative mechanical system consists of deformable and mass inertial elements connected by massless deformable elements, which is represented in the structural theory of the ejection protection systems by a structural scheme resolved with respect to the isolated object of protection with the transfer function of the integrating link, is expressed by partial differential equations of mathematical physics, then negative feedback, built on the basis of the geometric servo constraints there is at only when the vibration-insulated object is in a stable state.

The proof of this theorem is confirmed on numerous examples, which are solved by numerically and semianalytically methods. Thus, the feedback principle is the methodological basis of a new direction in the theory of oscillations and the application of the latter and the solution of problems of dynamics of controlled motion, in that part of them that is related to the protection of machinery and equipment from vibrations.

\section{ACtiVE VibRAtion PRotection OF THE MECHANICAL SYSTEM}

The main stages of the work are the creation of mathematical models of dynamic processes in the machines and constructions under investigation, as well as the search for controlled vibration damping.

Active vibration protection of a mechanical system, with a finite number of degrees of freedom. Let, the mechanical system consists of $\mathrm{N}$ masses connected by deformable elements. We confine ourselves to considering such oscillations, which are described by linear differential equations. In order for the equations of motion to be linear, it is necessary that the deviations of the system from the equilibrium position be sufficiently small (which is ensured by the smallness of the initial perturbations). Since it is required to cancel the oscillations of the system with respect to zero initial positions, we assume that the motion of the system is constrained by servo constraints [20]:

$q_{1}=0, q_{2}=0, \ldots, q_{n}=0$

where $q_{1}, q_{2}, \ldots, q_{n}$ - generalized coordinates, which describe the motion of a mechanical system.

Since the system oscillates with respect to the position (5), along with relations (5), the relations [21],[22]:

$$
q_{1}=\eta_{1}, q_{2}=\eta_{2}, \ldots, q_{n}=\eta_{n}
$$

where $\eta_{1}, \eta_{2}, \ldots, \eta_{n}{ }^{-} \quad$ independent parameters characterizing the release of the system from servo links (5).

The displacements, in which the reactions of servo constraints do not produce work [23], have the form:

$$
\delta q_{1}=0, \delta q_{2}=0, \ldots, \delta q_{n}=0
$$

or taking into account the relations (5):

$$
\delta \eta_{1}=0, \delta \eta_{2}=0, \ldots, \delta \eta_{n}=0
$$

Then the equations of motion of a system with factors can be written in the form:

$\frac{d}{d t}\left(\frac{\partial T}{\partial \dot{q}_{i}}\right)-\frac{\partial T}{\partial q_{i}}=\frac{\partial \Pi}{\partial q_{i}}+Q_{i}+\lambda_{i}$,

$(i=1, \ldots, n)$

where $_{\boldsymbol{T}, \boldsymbol{I}^{-}}$the kinetic and potential energies of the system;

$Q_{i}$ - generalized force corresponding to the generalized coordinate $q_{i} ; \quad \lambda_{i}$ - reaction forces of servo constraints.

In (6) we substitute the expressions for the kinetic and potential energies:

$$
T=\frac{1}{2} \sum_{i=1}^{n} \sum_{j=1}^{n} a_{i j} \dot{q}_{i} \dot{q}_{j}, \quad \Pi=\frac{1}{2} \sum_{i=1}^{n} \sum_{j=1}^{n} c_{i j} q_{i} q_{j},
$$

and after computing $\frac{\partial \boldsymbol{T}}{\partial \dot{\boldsymbol{q}}_{i}}, \frac{\partial \boldsymbol{T}}{\partial \boldsymbol{q}_{\boldsymbol{i}}}$, taking into account relations (2), we obtain:

$$
\left\{\begin{array}{l}
a_{11} \ddot{\eta}_{1}+a_{12} \eta_{2}^{\prime \prime}+\ldots+a_{1 n} \eta_{n}^{\prime \prime}+c_{11} \eta_{1}+c_{12} \eta_{2}+\ldots+c_{1 n} \eta_{n}=Q_{1}+\lambda_{1} \\
a_{21} \eta_{1}^{\prime \prime}+a_{22} \eta_{2}^{\prime \prime}+\ldots+a_{2 n} \eta_{n}^{\prime \prime}+c_{21} \eta_{1}+c_{22} \eta_{2}+\ldots+c_{2 n} \eta_{n}=Q_{2}+\lambda_{2} \\
\ldots \ldots \ldots \ldots \ldots \ldots \ldots \ldots \ldots \ldots \ldots \ldots \ldots \ldots \ldots \ldots \ldots \ldots \ldots \ldots \ldots \ldots \ldots \ldots \ldots \ldots \ldots \ldots \ldots \ldots \ldots \ldots \ldots \ldots \ldots \ldots \ldots \ldots \ldots \ldots \ldots \ldots \ldots \ldots \ldots \ldots \ldots \ldots \ldots \ldots \ldots \ldots \ldots \ldots \\
a_{n 1} \eta_{1}^{\prime \prime}+a_{n 2} \eta_{2}^{\prime \prime}+\ldots+a_{n n} \eta_{n}^{\prime \prime}+c_{n 1} \eta_{1}+c_{n 2} \eta_{2}+\ldots+c_{n n} \eta_{n}=Q_{n}+\lambda_{n}
\end{array}\right.
$$

These equations are differential equations of forced oscillations of a system with a finite number of degrees of freedom.

If we use the constructive method of finding the structure of the forces of the reactions of servo constraints [23],[24] and the reaction of the servo constraints $\lambda_{1}, \lambda_{2}, \ldots, \lambda_{n}$ form by law:

$$
\begin{aligned}
& \lambda_{1}=b_{11} \dot{\eta}_{1}+b_{12} \dot{\eta}_{2}+\ldots+b_{1 n} \dot{\eta}_{n}+d_{11} \eta_{1}+d_{12} \eta_{2}+\ldots+d_{1 n} \eta_{n}-Q_{1} \\
& \lambda_{2}=b_{21} \dot{\eta}_{1}+b_{22} \dot{\eta}_{2}+\ldots+b_{2 n} \dot{\eta}_{n}+d_{21} \eta_{1}+d_{22} \eta_{2}+\ldots+d_{2 n} \eta_{n}-Q_{2} \\
& \lambda_{n}=b_{n 1} \dot{\eta}_{1}+b_{n 2} \dot{\eta}_{2}+\ldots+b_{n n} \dot{\eta}_{n}+d_{n 1} \eta_{1}+d_{n 2} \eta_{2}+\ldots+d_{n n} \eta_{n}-Q_{n}
\end{aligned}
$$

where $b_{11}, b_{12}, \ldots, b_{n n} \quad d_{11}, d_{12}, \ldots, d_{n n}$ - some constant coefficients, then the equations of forced oscillations (5) of the system will have the form:

$$
\left\{\begin{array}{l}
a_{11} \ddot{\eta}_{1}+\ldots+a_{1 n} \ddot{\eta}_{n}+b_{11} \dot{\eta}_{1}+\ldots+b_{1 n} \dot{\eta}_{n}+\widehat{c}_{11} \eta_{1}+\ldots+\widehat{c}_{1 n} \eta_{n}=0 \\
a_{21} \ddot{\eta}_{1}+\ldots+a_{2 n} \ddot{\eta}_{n}+b_{21} \dot{\eta}_{1}+\ldots+b_{2 n} \dot{\eta}_{n}+\widehat{c}_{21} \eta_{1}+\ldots+\widehat{c}_{2 n} \eta_{n}=0 \\
\ldots \ldots \ldots \ldots \ldots \ldots \ldots \ldots \ldots \ldots \ldots \ldots \ldots \ldots \ldots \ldots \ldots \ldots \ldots \ldots \ldots \ldots \ldots \ldots \ldots \ldots \ldots \ldots \ldots \ldots \ldots \ldots \ldots \ldots \ldots \ldots \ldots \ldots \ldots \ldots \ldots \ldots \ldots \ldots \\
a_{n 1} \ddot{\eta}_{1}+\ldots \ldots+a_{n n} \ddot{\eta}_{n}+b_{n 1} \dot{\eta}_{1}+\ldots+b_{n n} \dot{\eta}_{n}+\widehat{c}_{n 1} \eta_{1}+\ldots+\widehat{c}_{n n} \eta_{n}=0
\end{array}\right.
$$

The system (7) has a particular solution:

$$
\eta_{1}=0, \eta_{2}=0, \ldots, \eta_{n}=0
$$

corresponding to position (1). Let us investigate the stability of the state (1). For this we formulate the characteristic determinant: 


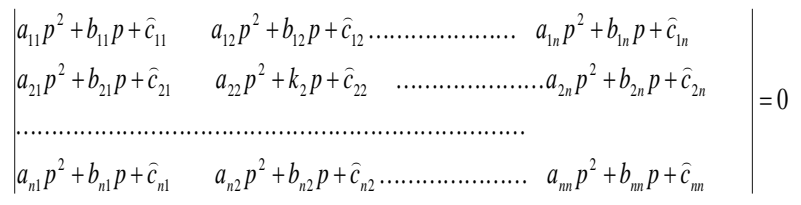

and revealing the determinant, we obtain the characteristic equation of the system (7):

$$
b_{1} p^{2 n}+b_{1} p^{2 n-1}+\ldots+b_{2 n-1} p+b_{2 n}=0
$$

here $b_{0}, b_{1}, \ldots b_{2 n}$ - positive constants depending on $a_{11}, a_{22}, \ldots . ., a_{n n}, b_{11}, b_{12}, \ldots, b_{1 n}, c_{11}, c_{22}, \ldots . c_{n n . .}$.

As is well known, for the stability of the particular solution (7) it is necessary and sufficient that all the roots of the characteristic equation of system (8) have negative real parts [25], [26]. Since the coefficients of (8) are constant numbers, the latter conditions can be obtained using the Hurwitz criterion [27],[28].

On the active vibration protection of a body having six degrees of freedom. Consider a body of mass $m$ suspended from the base of the shock absorbers. Let the body be imposed with a servo constraint [29]:

$$
\left.\begin{array}{ll}
q_{1}=0, & q_{2}=0, \quad q_{3}=0 \\
\varphi_{1}=0, & \varphi_{2}=0, \quad \varphi_{3}=0
\end{array}\right\}
$$

It is known [30], [31] that along with (9):

$$
\begin{aligned}
& q_{1}=\xi_{1}, \quad q_{2}=\xi_{2}, \quad q_{3}=\xi_{3}, \\
& \left.\varphi_{1}=\xi_{4}, \varphi_{2}=\xi_{5}, \varphi_{3}=\xi_{6}\right\}
\end{aligned}
$$

where $\zeta_{1}, \zeta_{2}, \ldots, \zeta_{6}{ }^{-}$independent parameters characterizing the continuous release of the system from servo constraints (9).Displacements, in which the reactions of servo constraints the work do not produce [30], have the form:

$$
\left.\begin{array}{l}
\delta \xi_{1}=0, \quad \delta \xi_{2}=0, \quad \delta \xi_{3}=0 \\
\delta \xi_{4}=0, \quad \delta \xi_{5}=0, \quad \delta \xi_{6}=0
\end{array}\right\}
$$

If we neglect the mass and damping of the shock absorbers, and the displacement of the body is assumed to be sufficiently small, then the motion of such a single mass system can be described by $\mathrm{n}$ second-order differential equations. Forming the equations in the form of Lagrange equations, we obtain:

$$
\begin{aligned}
& m \ddot{\xi}_{1}+\sum_{i=1}^{4} C_{x i} \xi_{1}+\sum_{i=1}^{4} C_{x i} z \cdot \xi_{5}-\sum_{i=1}^{4} C_{x i} y \cdot \xi_{6}=Q_{1} \cdot \cos \left(\Omega t+\psi_{1}\right)+\wedge_{1} \\
& m \ddot{\xi}_{2}+\sum_{i=1}^{4} C_{y i} \xi_{2}-\sum_{i=1}^{4} C_{y i} z \cdot \xi_{4}+\sum_{i=1}^{4} C_{y i} x \cdot \xi_{6}=Q_{2} \cdot \cos \left(\Omega t+\psi_{2}\right)+\wedge_{2} \\
& m \ddot{\xi}_{3}+\sum_{i=1}^{4} C_{z i} \xi_{3}+\sum_{i=1}^{4} C_{z i} y \cdot \xi_{4}-\sum C_{z i} x \cdot \xi_{5}=Q_{3} \cdot \cos \left(\Omega t+\psi_{3}\right)+\wedge_{3} \\
& I_{x} \ddot{\xi}_{4}-I_{x y} \cdot \ddot{\xi}_{5}-I_{z z} \ddot{\xi}_{6}-\sum_{i=1}^{4} C_{y i} \cdot z \cdot \xi_{2}+\sum_{i=1}^{4} C_{z i} y \cdot \xi_{3}+\sum_{i=1}^{4}\left(C_{z i} \cdot y^{2}-C_{y i} z^{2}\right) . \\
& \cdot \xi_{4}-\sum_{i=1}^{4} C_{z i} x y \cdot \xi_{5}-\sum_{i=1}^{4} C_{y i} x y \cdot \xi_{6}=M_{1} \cdot \cos \left(\Omega t+\psi_{4}\right)+\wedge_{4} \\
& I_{y} \ddot{\xi}_{5}-I_{x y} \cdot \ddot{\xi}_{4}-I_{y z} \ddot{\xi}_{6}+\sum_{i=1}^{4} C_{x i} z \cdot \xi_{1}-\sum_{i=1}^{4} C_{z i} x \cdot \xi_{3}-\sum_{i=1}^{4} C_{z i} x y . \\
& \cdot \xi_{4}+\sum_{i=1}^{4}\left(C_{x i} z^{2}+C_{z i} x^{2}\right) \cdot \xi_{5}-\sum_{i=1}^{4} C_{x i} y \cdot \xi_{6}=M_{2} \cdot \cos \left(\Omega t+\psi_{5}\right)+\wedge_{5}
\end{aligned}
$$

$$
\begin{aligned}
& I_{z} \cdot \ddot{\xi}_{6}-I_{x y} \ddot{\xi}_{4}-I_{y z} \ddot{\xi}_{5}-\sum_{i=1}^{4} C_{x i} y \cdot \xi_{1}+\sum_{i=1}^{4} C_{y i} x \cdot \xi_{2}-\sum_{i=1}^{4} C_{y i} x y . \\
& \cdot \xi_{4}-\sum_{i=1}^{4} C_{x i} z y \cdot \xi_{5}+\sum_{i=1}^{4}\left(C_{x i} y^{2}+C_{y i} x^{2}\right) \xi_{6}=M_{3} \cdot \cos \left(\Omega t+\psi_{3}\right)+\wedge_{6}
\end{aligned}
$$

where $\wedge_{1}, \wedge_{2}, \ldots, \wedge_{6}-$ servo constraint reactions, $I_{x}, I_{y}, I_{z}, \ldots$-moments of inertia, $\mathrm{C}_{\mathrm{x}}, \mathrm{C}_{\mathrm{y}}, \mathrm{C}_{\mathrm{z}}, .$. - Rigidity, $\mathrm{Q}_{1}$, $\mathrm{Q}_{2}, \mathrm{Q}_{3}, \mathrm{M}_{1}, \mathrm{M}_{2}, \mathrm{M}_{3}$ - amplitudes of external forces and moments.

Using the constructive method of finding the structure of the reaction forces of the servo constraints [30], [31], if the servo constraint reactions are formed according to the laws:

$$
\begin{aligned}
& \wedge_{1}=\sum_{i=1}^{4} C_{x i} \xi_{1}+\sum_{i=1}^{4} C_{x i} z \cdot \xi_{5}-\sum_{i=1}^{4} C_{x i} y \cdot \xi_{6} Q_{1} \cdot \cos \left(\Omega t+\psi_{1}\right)-k_{11} \dot{\xi}_{1}-k_{12} \xi_{1} \\
& \wedge_{2}=\sum_{i=1}^{4} C_{y i} \xi_{2}-\sum_{i=1}^{4} C_{y i} z \cdot \xi_{4}+\sum_{i=1}^{4} C_{y i} x \cdot \xi_{6}-Q_{2} \cdot \cos \left(\Omega t+\psi_{2}\right)-k_{21} \dot{\xi}_{1}-k_{22} \xi_{2} \\
& \wedge_{3}=\sum_{i=1}^{4} C_{z i} \xi_{3}+\sum_{i=1}^{4} C_{z i} y \cdot \xi_{5}-\sum_{i=1}^{4} C_{z i} x \cdot \xi_{5}-Q_{3} \cdot \cos \left(\Omega t+\psi_{3}\right)-k_{31} \dot{\xi}_{3}-k_{32} \xi_{3} \\
& \wedge_{5}=-I_{x y} \ddot{\xi}_{4}-I_{y z} \ddot{\xi}_{6}+\sum_{i=1}^{4} C_{x i} z \cdot \xi_{1}-\sum_{i=1}^{4} C_{z i} x \cdot \xi_{3}-\sum_{i=1}^{4} C_{z i} x y \cdot \xi_{4}+\sum_{i=1}^{4}\left(C_{x i} z^{2}+C_{z i} x^{2}\right) \cdot \xi_{5}- \\
& \sum_{i=1}^{4} C_{x i} y \cdot \xi_{6}-M_{2} \cos \left(\Omega \cdot t+\psi_{5}\right)-k_{51} \cdot \dot{\xi}_{5}-k_{52} \cdot \xi_{5} \\
& \wedge_{6}=-I_{x y} \cdot \ddot{\xi}_{4}-I_{y z} \ddot{\xi}_{5}-\sum_{i=1}^{4} C_{x i} y \cdot \xi_{1}+\sum_{i=1}^{4} C_{y i} x \cdot \xi_{2}-\sum_{i=1}^{4} C_{y i} x y \cdot \xi_{4}- \\
& \sum_{i=1}^{4} C_{x i} z y \cdot \xi_{5}+\sum_{i=1}^{4}\left(C_{x i} y^{2}+C_{y i} x^{2}\right) \cdot \xi_{6}-M_{3} \cos \left(\Omega t+\psi_{6}\right)-k_{61} \dot{\xi}_{6}-k_{62} \xi_{6},
\end{aligned}
$$

where $k_{11}, k_{12}, \ldots, k_{61}, k_{62}$ - some constants, then substituting (13) into (12), we obtain the equations of disturbed motion:

$$
\left\{\begin{array}{l}
m \xi_{1}+k_{11} \dot{\xi}_{1}+k_{12} \xi_{1}=0 \\
m \xi_{2}+k_{21} \xi_{2}+k_{22} \xi_{2}=O \\
m \dot{\xi}_{3}+k_{31} \dot{\xi}_{3}+k_{32} \xi_{3}=O \\
I_{x} \cdot \xi_{4}+k_{41} \dot{\xi}_{4}+k_{42} \xi_{4}=O \\
I_{y} \cdot \dot{\xi}_{5}+k_{51} \dot{\xi}_{5}+k_{52} \xi_{5}=O \\
I_{z} \cdot \xi_{6}+k_{61} \dot{\xi}_{6}+k_{62} \xi_{6}=O
\end{array}\right.
$$

The system (14) has a particular solution:

$$
\begin{aligned}
& \xi_{1}=0, \quad \xi_{2}=0, \quad \xi_{3}=0 \\
& \xi_{4}=0, \quad \xi_{5}=0, \quad \xi_{6}=0 .
\end{aligned}
$$

Corresponding to constraints (9).

As is known, for the stability of the particular solution (15) it is necessary and sufficient that all the roots of the characteristic equation of system (14) have negative real parts [32], [33]. Since the coefficients of (14) are constant numbers, the latter conditions can be obtained using the Hurwitz criterion [28]. We investigate for what values of the constants $k_{11}, k_{12}, \ldots, k_{61}, k_{62}$ the stability of the zero solution (15) of the system (14) is ensured. To do this, we formulate the characteristic determinant of the system (14):

$$
\left|\begin{array}{ccccccc}
m \lambda^{2}+\kappa_{11} \lambda+\kappa_{12} & 0 & 0 & 0 & 0 & 0 \\
0 & m \lambda^{2}+\kappa_{21} \lambda+\kappa_{22} & 0 & 0 & 0 & 0 \\
0 & 0 & m \lambda^{2}+\kappa_{31} \lambda+\kappa_{32} & 0 & 0 & 0 \\
0 & 0 & 0 & I_{x} \lambda^{2}+\kappa_{41} \lambda+\kappa_{42} & 0 & 0 \\
0 & 0 & 0 & 0 & I_{y} \lambda^{2}+\kappa_{51} \lambda+\kappa_{52} & 0 \\
0 & 0 & 0 & 0 & 0 & I_{z} \lambda^{2}+\kappa_{61} \lambda+\kappa_{62}
\end{array}\right|
$$


The characteristic equation of the system has the form:

$$
\begin{array}{r}
\left(m \lambda^{2}+\kappa_{11} \lambda+\kappa_{12}\right)\left(m \lambda^{2}+\kappa_{21} \lambda+\kappa_{22}\right) \\
\left(m \lambda^{2}+\kappa_{31} \lambda+\kappa_{32}\right)\left(I_{x} \lambda^{2}+\kappa_{41} \lambda+\kappa_{42}\right) \\
\left(I_{y} \lambda^{2}+\kappa_{51} \lambda+\kappa_{52}\right)\left(I_{z} \lambda^{2}+\kappa_{61} \lambda+\kappa_{62}\right)=0
\end{array}
$$

The roots of the characteristic equation (16):

$$
\begin{gathered}
\lambda_{i}=\frac{-k_{i 1} \pm\left(k_{i 1}^{2}-4 \cdot m \cdot k_{i 2}\right)^{1 / 2}}{2 \cdot m}, \\
\lambda_{3+i i, j}=\frac{-k_{3+i, 1} \pm\left(k_{3+i, 1}^{2}-4 \cdot I \cdot k_{3+i, 2}\right)^{1 / 2}}{2 \cdot I}, \quad(i=1,2,3 ; \mathrm{j}=1,2)
\end{gathered}
$$

We choose them so that, they have negative real parts; the conditions:

$$
\left.k_{i 1}>0, \quad k_{i 2}>0, \quad k_{3+i, 1}>0, k_{3+i, 2}\right\rangle 0,(i=1,2,3),
$$

which are achieved by a suitable choice of parameters $m$, $I_{x}, I_{y}, I_{z}$ system and permanent $\kappa_{11, \ldots,} \kappa_{62}$. The conditions (17) express the conditions for the asymptotic stability of the positions (15).

Active dynamic damping of oscillations of a system with one degree of freedom. We consider a load reinforced on a spring with rigidity ${ }_{C_{1}}$, which makes forced oscillations under the action of a disturbing force

$$
Q=Q_{\mathrm{o}} \cdot \sin p t
$$

where $Q_{0}$-amplitude; $p$-frequency; $t$-time, or base vibration by law

$$
x_{0}(t)=x_{0} \sin p t
$$

here $x_{0}$-amplitude. Under the action of the applied perturbation, the object performs one-dimensional oscillations with amplitude [34]:

$$
a=\frac{Q_{0}}{C_{1}\left[1-\left(\frac{\omega}{\omega_{0}}\right)^{2}\right]}
$$

where $\omega_{0}=\sqrt{\frac{c_{1}}{m}}$-the natural frequency of the object. When $\omega \rightarrow \omega_{0}$ the fluctuations of the object increase substantially. It is required to determine under what conditions these oscillations can be quenched by attaching to the load 1 on a spring with a stiffness coefficient ${ }_{C_{1}}$ and a viscous dampener with a viscosity coefficient ${ }_{b_{r}}$ cargo 2 tons $m_{\Gamma}$. This means that one servo link is imposed on the "cargo-damper" system [21,22]:

$$
\boldsymbol{x}=\mathbf{O}
$$

Since the force of the servo constraint reaction acts on the absorber, the (A) -movings [35] will have the form:

$$
\delta x_{\Gamma}=\mathrm{O}
$$

Since the absorber is a body of variable mass, the general equation of dynamics should be taken in the form $[35,36]$ :

$$
\sum_{i=1}^{3}\left(\frac{d^{*}}{d t} \frac{\partial^{*} T}{\partial \dot{q}_{i}}-\frac{\partial^{*} T}{\partial q_{i}}\right) \delta q_{i}=\sum_{i=1}^{3}\left(Q_{i}+R_{i}\right) \delta q_{i}
$$

where for $q_{i}$ the coordinates ${ }_{x_{\Gamma}}, x ; Q_{i}-$ generalized force referred to the coordinate $q_{i} ; R_{i}-$ generalized reactive force, which has the form:

$$
R_{i}=\sum_{v=1}^{n}\left[\frac{d m_{v}}{d t}\left(\bar{U}_{v}-\bar{v}_{v}\right)-m_{v} \bar{a}_{v}^{r}\right] \frac{d \bar{r}_{v}}{d q_{i}}
$$

here $\left(\bar{U}_{v}-\bar{v}_{v}\right)$ - the relative velocity of particles acceding; $\bar{l}_{v}^{\prime}$ - acceleration of the particle in motion relative to the coordinate system associated with the constraint, and the $\operatorname{sign} *$ at the top indicates that the differentiation is made in accordance with the principle of solidification. Then the differential equations of oscillations of the system with a quenching have the following form:

$$
\left\{\begin{array}{l}
m \xi+b_{\Gamma}\left(\dot{\xi}-\dot{x}_{\Gamma}\right)+C_{1} \xi+C_{\Gamma}\left(\xi-x_{\Gamma}\right)=Q_{0} \sin p t \\
m_{\Gamma} \ddot{x}_{\Gamma}+b_{\Gamma}\left(\dot{x}_{\Gamma}-\dot{\xi}\right)+C_{\Gamma}\left(x_{\Gamma}-\xi\right)=R_{2}
\end{array}\right.
$$

where $\boldsymbol{R}_{2}$ - servo constraint force.

It is known [37]-[39] that along with relations (18):

$$
x=\xi
$$

where $\xi$-release parameter from servo constraint. Zero value $\xi$ corresponds to the relation (18) or its differentiated form.

Let's set the task: to determine by what law to regulate the force of the servo constraint reaction $R_{2}$, so that the servo constraint (18) is stable. Writing equations (20) in the form:

$$
\begin{gathered}
\left(m_{\Gamma} \ddot{\xi}+b_{\Gamma} \dot{\xi}+C_{\Gamma} \xi\right)-\left(b_{\Gamma} \dot{x}_{\Gamma}+C_{\Gamma} x_{\Gamma}\right)=Q_{0} \sin p t \\
\left(m_{\Gamma} \ddot{x}_{\Gamma}+b_{\Gamma} \dot{x}_{\Gamma}+C_{\Gamma} x_{\Gamma}\right)-\left(b_{\Gamma} \dot{\xi}+C_{\Gamma} \xi\right)=R
\end{gathered}
$$

from the second equation of system (22) we define expression $\left(b_{\Gamma} \dot{x}_{\Gamma}+C_{\Gamma} x_{\Gamma}\right)$, т.e.,

$$
\left(b_{\Gamma} \dot{x}_{\Gamma}+C_{\Gamma} x_{\Gamma}\right)=-m_{\Gamma} \ddot{x}_{\Gamma}+b_{\Gamma} \dot{\xi}+C_{\Gamma} \xi+R .
$$

Substituting (23) into the first equation of system (22), we obtain:

$$
m_{\Gamma} \ddot{\xi}+\left(C_{1}-C_{\Gamma}\right) \xi+m_{\Gamma} \ddot{x}_{\Gamma}-Q_{0} \sin p t=R
$$

Hence, if the servo constraint reaction is formed by law: 


$$
R=m_{\Gamma} \ddot{x}_{\Gamma}-Q-k_{1} \dot{\xi}-k_{2} \xi
$$

where $k_{1}, k_{2}$ - some constants, then, substituting (25) in (24), we obtain the equations of the disturbed motion of the object:

$$
m_{\Gamma} \ddot{\xi}+k_{1} \dot{\xi}+\left(C_{1}-\tilde{N}_{\tilde{A}}+k_{2}\right) \xi=0
$$

Equation (26) is a differential equation with constant coefficients. Let us investigate the stability of an undisturbed motion

$$
\xi=\mathbf{O}
$$

the corresponding servo constraint (18). To do this, we formulate the characteristic equation, which has the form:

$$
m_{\tilde{A}} \lambda^{2}+\left(k_{1}+\frac{d m_{\Gamma}}{d t}\right) \lambda+\left(C_{1}+k_{2}\right)=0 .
$$

Equation (27) is a quadratic equation with constant coefficients. Therefore, the stability of the implementation of servo-constraints (the negativity of the real parts of the roots of the characteristic equation) can be investigated by the Hurwitz criterion [28]. For this we formulate the characteristic determinant (Hurwitz Ian):

$$
\Delta=\left|\begin{array}{lll}
m_{\tilde{A}} & C_{1}+k_{2} & 0 \\
0 & \left(k_{1}+\frac{d m_{\Gamma}}{d t}\right) & 0 \\
0 & m_{\tilde{A}} \quad C_{1}+k_{2}
\end{array}\right| .
$$

According to the Hurwitz theorem, the necessary and sufficient condition for the negativity of the real parts of the roots of the characteristic equation (the asymptotic stability condition) for the equation of the second degree will have the form [28]:

$$
\begin{gathered}
m_{\tilde{A}}>0 \quad, \quad k_{1}+\frac{d m_{\Gamma}}{d t}>0 \quad, \quad \Delta_{1}=m_{\Gamma}>0 \\
\Delta_{2}=\left|\begin{array}{ccc}
m_{\tilde{A}} & C_{1}+k_{2} \\
0 & k_{1}+\frac{d m_{\Gamma}}{d t}
\end{array}\right|=m_{\tilde{A}} \cdot\left(k_{1}+\frac{d m_{\Gamma}}{d t}\right)>0 \\
\Delta_{3}=\left|\begin{array}{lll}
m_{\tilde{A}} & C_{1}+k_{2} & 0 \\
0 & k_{1}+\frac{d m_{\Gamma}}{d t} & 0 \\
0 & m_{\tilde{A}} & C_{1}+k_{2}
\end{array}\right|=m_{\tilde{A}} \cdot\left(k_{1}+\frac{d m_{\Gamma}}{d t}\right) \cdot\left(C_{1}+k_{2}\right)>0
\end{gathered} .
$$

those. The Hurwitz conditions are reduced to inequalities:

$$
m_{\tilde{A}}>0 \quad, \quad k_{1}+\frac{d m_{\Gamma}}{d t}>0, \quad C_{1}+k_{2}>0
$$

\section{EVALUATION OF THE EFFECTIVENESS OF THE APPLICATION OF THE RANGE OF A CONTROLLED VIBRATION PROTECTION SYSTEM}

Transfer function and its definitions. At present, there is a sufficiently large number of ejection means, with adjustable parameters, the structural diagram of which is shown in Fig. 3 . In the general case, three main parts can be distinguished in the design scheme: the source of the disturbance, the object of protection, and that part of the mechanical oscillatory system that performs the functions of reducing the level of impacts. For further consideration, we choose translational motions along a certain axis $q$. The vibration isolator is the most important part of the vibration protection system, its purpose is to create such a mode, a motion triggered by predetermined disturbances, in which the object protection objective is realized.

In many cases, this is achievable by using an inertia-free vibration isolation device, depicted in Fig. 3. For such a vibration isolator $\vec{R}_{\vec{R}}$ and $_{\vec{R}^{\prime}}$ coincide in magnitude $(|\vec{R}|=|\vec{R}|$ ).Thus, in the simplest case, the reaction can be considered proportional to the mixing or mixing speed of the object

$$
\vec{R}=[K] \vec{q}+[B] \frac{d q}{d t},
$$

where $[K]$ - square matrix of rigidity, $[B]$ - square damping matrix. The reaction forces (29) can be described using the Boltzmann-Voltaire integral

$$
\vec{R}=\left[K_{\mathrm{o}}\right]\left\{\vec{q}(t)-\int_{-\infty}^{t}[R(t-\tau)] \vec{q}(\tau) d \tau\right\},
$$

where $\left[K_{0}\right]$-instantaneous stiffness matrix, $[R(t-\tau)]$-matrix of rheological properties without mass deformable elements.

Adjustment of the system parameters is carried out according to the laws, proceeding from the given requirements to the vibration protection. Dependence (29) describes the linear characteristic of a simple inertial system of vibration isolation. When $n_{[B]}=0$ protected objects are connected to each other and the base by elastic elements (springs), and when $\left[K_{0}\right]=0$ the objects to be protected are connected to each other and to the base by viscous dampers.

Under the effectiveness of vibration protection, we understand the degree of implementation of vibration protection devices. With force harmonic action, the force varies according to law:

$$
P(t)=P_{0} \sin \omega t,
$$

where $P_{0}$ and $\boldsymbol{\omega}$ - respectively, the amplitude and frequency of the driving force. The purpose of protection can be to reduce the amplitude $\vec{R}_{0}$ - force transferred to a stationary object. As an example, consider a mechanical system with one degree of freedom (Fig. 3). Here

$$
R_{\mathrm{o}}=\frac{P_{o} \sqrt{\omega^{4}+4 n^{2} \omega}}{\sqrt{\left(\omega_{o}^{2}-\omega^{2}\right)^{2}+4 n^{2} \omega^{2}}},
$$

where, $n=b /(2 m)$, b- coefficient of damping. Using Laplace transforms [40], we find that $R_{0}=\bar{q}(b p+k)$. In turn, the decrease in the oscillations of the object under the action of the force is determined by the formula: 


$$
q_{0}=\frac{P_{o}}{m \sqrt{\left(\omega_{o}^{2}-\omega^{2}\right)^{2}+4 n^{2} \omega^{2}}} .
$$

Transfer function of the system with input signal ${ }_{\bar{P}}$ and the output signal $\vec{R}_{0}$ has the form

$$
W_{b}(p)=\frac{\bar{R}_{0}}{\bar{P}}=\frac{b p+k}{m p^{2}+b p+k} .
$$

When designing vibration protection systems, they tend to ensure that the natural frequency of the system is as small as possible. In this case, the frequency band of effective vibration isolation expands. However, a decrease in the frequency of natural oscillations due to a decrease in the rigidity of the elastic elements or an increase in the mass of the object is not always possible because of the large static shrinkage of the elastic elements. Some possibilities in this respect are possessed by protection systems with additional passive connections with respect to the relative deviation, the design model of which is shown in Fig. 1a, and the structural scheme is shown in Figure. In this scheme $L(p)$ - the transfer function of the additional connection (the latter can have a different physical nature) [41]. Transfer function of the system

$$
W(p)=\frac{c_{o} p+k_{o}+L(p)}{m p^{2}+c_{o} p+k_{o}+L(p)},
$$

where $k_{o}, c_{o}, m$ - respectively, the stiffness, damping and mass of the object-the parameters of the original passive vibration protection system shows that the dynamic properties of the conventional vibration protection system are modified by an appropriate choice $L(p)$. Let's consider features of construction of active outtake protection systems having additional circuits in which control actions on absolute deviation of a protection object.

Dynamic model of a two-mass active vibration protection system (Fig. 3). The most effective way to combat vibration is to reduce the variable forces in sources and chains of energy transfer (internal combustion engines, gears, electric motors, etc.). But, naturally, when designing the sources, it is crucial to fulfill the main functional task - to ensure the transfer of energy from the source to the receiver with the maximum efficiency. with the obligatory fulfillment of the requirements for strength and resource characteristics. Vibration activity often recedes into the background. Hence the limited nature of this way of dealing with vibration.

To protect technical and biological objects from vibration excitation in the low frequency region, a huge number of vibration protective systems based on the use of a wide range of shock absorbers have now been developed. Such vibrato protective systems are called passive. However, their application in many cases is ineffective, for example, when protecting objects from time-varying vibration spectra.

Recently, automated vibration protection systems, called active [41],[42], have found application. The creation of effective active vibration damping systems for lowfrequency vibration of various mechanisms excited by the action of variable forces is the goal of the work of many researchers over the past several decades. In general, the management of such systems can be implemented on the principle of compensation of the disturbance, compensation of the deviation of the regulated quantity, or on a combination of both these methods.

The experience of creating active vibration damping systems has shown that the most promising in the sense of the completeness of the reproduction of variable forces, the comparative simplicity of implementation and control, and the lack of sensitivity to negative environmental factors are electrodynamics VZS, in which the electrodynamic vibrator serves as an executive device [43]. A characteristic feature of active systems is the use of active circuits consisting of measuring, amplifying and actuating elements. The latter form a force, allowing to reduce the dynamic loads acting on the protected object.

In active emission protection systems, to create a control action (control), information is needed on the nature of the perturbations, its frequency and amplitude composition. The role of the sources of this information is performed by electrical vibration transducers, which act as converters of the parameters of motion (force, acceleration, displacement) into electrical signals (voltage, current). The transducers used (displacement sensors, forces, accelerometers, etc.) should have a sufficiently wide frequency range (at least five times wider than the frequency range of the measured signal) and a low nonlinear distortion factor. Electrical signals as control actions should be proportional to the disturbing force $Q(t)$. When the frequency and the amplitude of the external action are varied, the frequency and amplitude of the current (voltage) should change in a similar way. The oscillating system with an electrodynamics vibrator can be regarded as an object of automatic control and apply methods developed in the theory of automatic control for its investigation [43], [44]. When compiling a system of equations describing the dynamics of a mechanical oscillatory system with an electrodynamics force generator, both mechanical motion and electrical processes in the conductor chain (movable coil) should be taken into account. In electrodynamics devices, the current for creating a force arises from the motion of either the conductor itself or its suspension points.

We will carry out a study of models of active ejection protection systems with various circuits for connecting an electrodynamics vibrator and applying an external dynamic load. In all cases, the task is to reduce the dynamic load on the foundation. Simulation is performed in the low frequency region. Consider the depiction in Fig. 4 model of active outtake protection systems.

Equations of motion of the system model of active outtake protection systems under assumptions - the system makes unidirectional movements - the movements of the masses $m_{1}$ and $m_{2}$ are considered with respect to the equilibrium position - has the following form (1) - (3), then the matrix of coefficients takes the following form

$$
[M]\left\{\frac{\partial^{2} X}{\partial t^{2}}\right\}+[B]\left\{\frac{\partial X}{\partial t}\right\}+[C]\{X\}=\{F(t)\} .
$$


To assess the effectiveness of the system of vibration isolation as an example, a system with parameters: $m_{1}=50$ $\mathrm{kg}, m_{2}=5 \mathrm{~kg}, m_{3}=1 \mathrm{~kg}, c_{1}=4.5 \cdot 10^{4} \mathrm{H} / \mathrm{M}, c_{2}=8 \cdot 10^{3} \mathrm{H} / \mathrm{M}, b_{0}$ $=35,5 \mathrm{HM} / \mathrm{c}, b_{1}=6,5 \mathrm{HM} / \mathrm{c}, c_{2}=350 \mathrm{H} / \mathrm{M}, b_{2}=6 \mathrm{HM} / \mathrm{c}, B_{l}=$ 10 Тл $\cdot \mathrm{M} ; L=5 \cdot 10^{-3} \Gamma \mathrm{H} ; R=10$ Ом; $K_{\text {дп }}=1 \mathrm{~B} / \mathrm{M} ; K_{1}=500$.

When choosing the feedback amplification factors $K_{0}$, $K_{1}$ It is necessary to consider both the stability of the servo system and the automatic control system as a whole. Tracking system with $K_{1} \geq 2 \cdot 10^{4}$ becomes unstable. Estimation of the stability of the automatic control system in accordance with (32) in the program

$$
W_{u_{s x} x_{2}}(p)=\frac{K_{1} B \ell}{h_{3} p^{3}+h_{2} p^{2}+h_{1} p+h_{0}},
$$

MAPLE-18 shows that with these parameters, stability is disrupted when $K_{l} \geq 1100$ (a complex root of the characteristic equation with a positive real part appears). Transmission unction $W_{u_{e x}}(p)$ for active vibration protection systems will have the form (33), where

$h_{3}=L m_{3}, \mathrm{~h}_{2}=L b_{3}+R m_{3}, \mathrm{~h}_{1}=L c_{3}+b_{2} R+(B \ell)^{2}, \mathrm{~h}_{0}=R c_{3}+K_{1} K_{\mathrm{dp}} \cdot B \ell$

The condition of stability by the Hurwitz criterion is the inequality:

$$
\left(L b_{2}+R m_{2}\right)\left(L c_{2}+b_{2} R+B^{2} \ell^{2}\right)>L m_{2}\left(R c_{2}+K_{1} K_{\text {дп }} \cdot B \ell\right) .
$$

The indicator that determines the effectiveness of the active system in relation to the passive system is the active vibration isolation coefficient $\gamma$ :

$$
\gamma=\frac{\overline{\boldsymbol{R}}_{\Phi}(p)}{\overline{\boldsymbol{R}}_{\Phi \mathrm{O}}(p)}
$$

The condition for effective active vibration isolation is the inequality $\gamma>1$. Substituting now $p=i \omega(\omega$-frequency of oscillations), we obtain a frequency dependence for the coefficient of vibration isolation. Fig. 4 shows the Dependence $\bmod \gamma(\omega)$ from $\omega$.

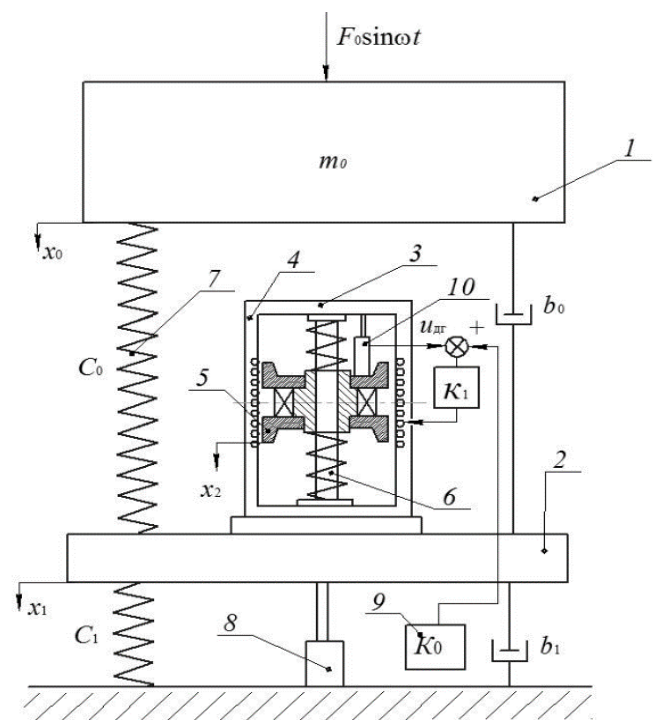

Fig. 3. Model of a two-mass mechanical system 1 - vibrating active mass $m_{0} ; 2$-intermediate frame with mass $m_{1} ; 3$ - electrodynamics compensator housing; 4 - excitation coil; 5 - permanent magnet with magnet wire and ground $m_{2} ; 6$ - guide; 7 - a spring; 8 - displacement sensor; 9 - amplifier; 10 - displacement sensor; $c_{0}, b_{0}, c_{1}, b_{1}$ - the stiffness and damping coefficients of the respective first and second stages; $x_{0}, x_{1}-$ absolute mass displacements $m_{0}$ and $m_{1} ; F_{0} \sin \omega t$ - vibrato active force.

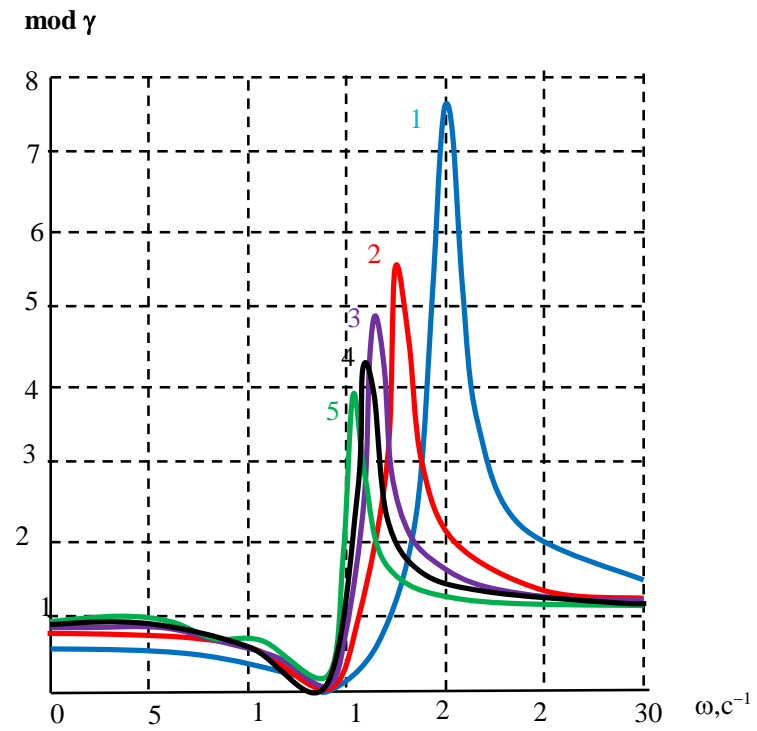

Fig. 4. Curves of the coefficient $\gamma$ of frequency $\omega$, constructed for different values of the coefficient of viscosity: the curve $1-b_{1}=0.05 ; 2-b_{1}=0.5 ; 3$ $-b_{1}=5 ; 4-b_{1}=10 ; 5-b_{1}=20$.

Let us investigate the stability of this system using the Hurwitz criterion. The characteristic equation of system (33) will have the form:

$\Delta(p)=\left|\begin{array}{ccc}\lambda_{p 1} & -\lambda_{12}^{2} & \rho_{1} \\ -\lambda_{2}^{2} & \lambda_{p 2} & -\rho_{2} \\ \lambda_{p 3} & \mu p & \lambda_{T}\end{array}\right|=0$

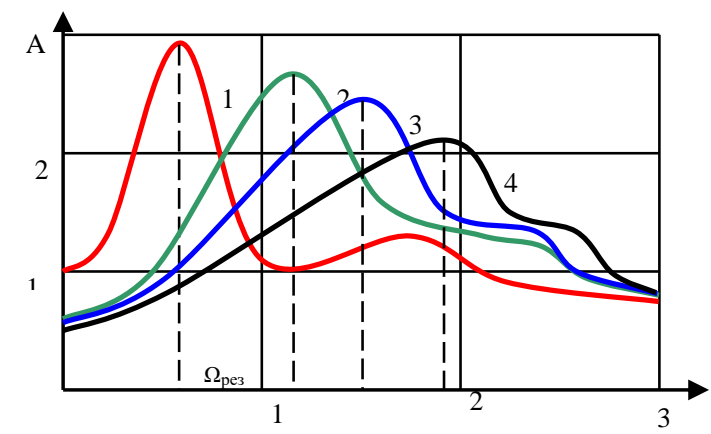

Fig. 5. Amplitude-frequency characteristics of active vibration protection systems with proportional control $\left(k_{a}\right)$ : 1 - amplitude - frequency

characteristics of the passive system; 2,3,4 - AFC of the active system in order of increasing the gain $\boldsymbol{K}_{\boldsymbol{a}}$

where $\lambda_{p 1}=p^{2}+p \beta_{1}+\lambda_{1}^{2}+\lambda_{2}^{2}, \lambda_{p 2}=p^{2}+p \beta_{2}+\lambda_{2}^{2}$,

$\lambda_{p 3}=k_{1} \mu p+k_{2}, \lambda_{T}=\boldsymbol{Z}, \boldsymbol{1} \mathbf{1}$.

Expanding the determinant, we obtain the equation of the fifth power in $p$ :

$b_{0} p^{5}+b_{1} p^{4}+b_{2} p^{3}+b_{3} p^{2}+b_{4} p+b_{5}=0$

where the coefficients $b_{j}(j=0,1,2,3,4,5)$ have the form: 
$b_{o}=T_{9}, b_{1}=T_{9}\left(\beta_{1}+\beta_{2}\right)+1, b_{2}=T_{9}\left(\lambda_{1}^{2}+2 \lambda_{2}^{2}+\beta_{1} \beta_{2}\right)-\rho_{1}\left(k_{1}-\mu\right)+\rho_{2} \mu+\beta_{1}+\beta_{2}$,

$b_{3}=T_{3} \beta_{2}\left(\lambda_{1}^{2}+\lambda_{2}^{2}\right)+T_{3} \beta_{1} \lambda_{2}^{2}-\rho_{1}\left(k_{1}-\mu\right) \beta_{2}-\rho_{1} k_{2}+\rho_{2} \beta_{1} \mu+\lambda_{1}^{2}+2 \lambda_{2}^{2}+\beta_{1} \beta_{2}$,

$b_{4}=T_{3} \lambda_{2}^{2}\left(\lambda_{1}^{2}+\lambda_{2}^{2}\right)-\rho_{1} \lambda_{2}^{2} \mu-\rho_{1}\left(k_{1}-\mu\right) \lambda_{2}^{2}-\rho_{1} \beta_{2} k_{2}+$

$+\left(\rho_{2} \mu+\beta_{2}\right)\left(\lambda_{1}^{2}+\lambda_{2}^{2}\right) \mu-T_{3} \lambda_{2}^{2} \lambda_{12}^{2}+\beta_{1} \lambda_{2}^{2}+\rho_{2}\left(k_{1}-\mu\right) \lambda_{12}^{2}$,

$b_{5}=\rho_{2} \lambda_{12}^{2} k_{2}-\rho_{1} \lambda_{2}^{2} k_{2}+-\rho_{1} \beta_{2} k_{2}+\lambda_{2}^{2}\left(\lambda_{1}^{2}+\lambda_{2}^{2}\right)-\lambda_{1}^{2} \lambda_{2}^{2}$.

In Fig. 6 shows the frequency characteristics

$\kappa_{\text {п }}(\omega)=\frac{|R(i \omega)|}{|F(i \omega)|}$

where $R(i \omega)=\left(c_{1}+b_{1} i \omega\right) x_{1}$, from which it follows that when installing the EHD on an intermediate platform, the frequency range of effective vibration isolation increases significantly and the effect of additional resonance on the transfer of force to the base decreases. For the parameters of the vibration isolation system under consideration $K_{0}=1000$ the system is effective in $f_{6 \mathrm{H}}>2 \mathrm{~Hz}$ (at resonance frequencies $f_{0}>3 \mathrm{~Hz}, f_{1}>5 \mathrm{~Hz}$ ).

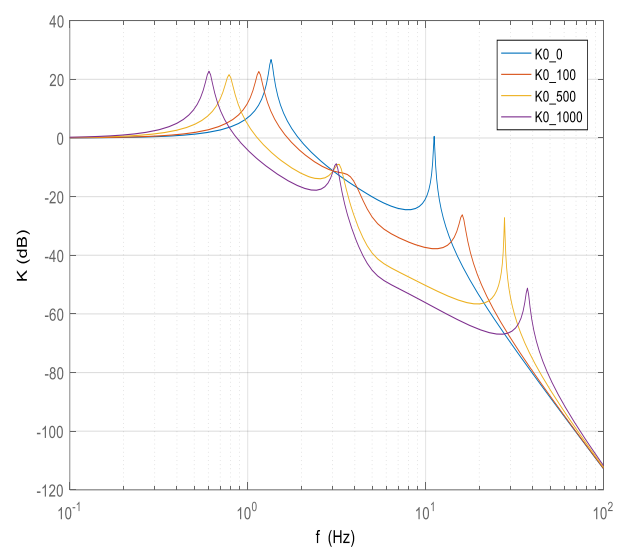

Fig. 6. Frequency characteristics

The Hurwitz criterion, in this case [44], reduces to the following inequalities:

$$
\begin{aligned}
& b_{j}>0, j=0 \ldots .5 \\
& \Delta_{2}=b_{1} b_{2}-b_{0} b_{3}>0, \\
& \Delta_{2}=\left(b_{1} b_{2}-b_{0} b_{3}\right)\left(b_{3} b_{4}-b_{2} b_{5}\right)-\left(b_{1} b_{4}-b_{0} b_{5}\right)^{2}>0 .
\end{aligned}
$$

here $\Delta_{2}, \Delta_{4}-$ the Hurwitz determinants of the second and fourth orders, corresponding to the characteristic equation of the fifth degree. The regions of stability numerically constructed from (36) and (37) are shown in Fig. 5. The values of the other parameters are the same as in Fig. 6 . When $k_{U}>4.2$, the system is unstable. It can be seen from the calculations that the ABCD corresponding to the model under consideration is highly effective (up to $\gamma=8$ ) and is stable in the frequency range $15 \div 25 c^{-1}$. Expansion of this range is possible by increasing the feedback coefficient $k U$, however, the system loses stability. The increase in magnetic flux B makes it possible to expand the frequency range of efficiency, but it significantly reduces the efficiency of $\bmod (\gamma)$.

\section{CONCLUSIONS}

The first developed a calculation technique and an algorithm for the application of geometric servo constraints for active control of oscillations of linear dissipative mechanical systems consisting of solid and deformable bodies, vibration protection of objects:

- the use of DC motors found the laws governing the formation of control actions that ensure the stability of the system with respect to the variety determined by servo constraints;

- for the dynamic mode, the task of determining control actions is reduced to solving the system of finite-difference equations by the Seidel iteration method;

- alternating current motors, for the stationary mode, an analytical law for the formation of control actions is found that ensures the stability of the system with respect to the manifold determined by servo constraints. For the dynamic regime, the problem is reduced to solving a system of finite-difference equations, which is solved by Seidel's method;

- developed a new method for implementing servo connections by electromechanical forces.

\section{REFERENCES}

[1] D.S. Steinberg, Vibrations analysis for electronic equipment, NewYork, 1973.

[2] Vibration in engineering: Handbook: In 6 vol. Moscow, Mechanical Engineering, 1978

[3] A.E. Kolesnikov, Noise and Vibration: A Textbook. Moscow, Shipbuilding, 1988.

[4] E.N.Makvetov, A.M. Tartakovsky, Mechanical influences andprotection of electronic equipment: Proc. for universities. Moscow, Radio and communication,1993.

[5] A. Bozhko, Passive and active vibration protection of ship mechanisms, Moscow, Shipbuilding, 1987.

[6] M. Amabili, R. Garziera, S. Carra. " Rotary inertia of added masses on vibrations of empty and fluid-filled circular cylindrical shells", Journal of Fluids and Structures, Vol. 21, 2005, No. 5 - 7, pp. 449458

[7] K. V. Avramov, F. Pellicano "Dynamical instability of cylindrical shell with big mass at the end", Reports of the National Academy of Sci ence of Ukraine, № 5, p. 41 - 46, 2006.

[8] R Chen. "Vibration of cylindrical panels ferrying a concentrated mass”, J. Applied Mechanics (Trans. ASME, Ser. E), Vol. 37, № 3, pp. $874-875,1979$.

[9] N. J. Mallon." Dynamic stability of a base-excited thin orthotropic cylindrical shell with top mass: simulations and experiments", Journal of Sound and Vibration, 329, pp. 3149 - 3170, 2010.

[10] K. Marguerre. "Theorie der gekrummten Platte grosser Formanderung", Proc. 5th Int. Congr. Appl. Mech., Cambridge, Mass., 1938. - New York: J. Willey and Son, 1939. pp. 93 - 101.

[11] V.A. Frolov. Mechanical effects and protection of electronic equipment. Proc. manual for universities, Moscow: Radio and Communication, 1984

[12] O.P. Gludkin. Methods and devices for testing RES and EMU. Moscow, Higher education school. 2001.

[13] Tests of electronic, electronicequipment and testing equipment, Ed A.I. Korobov, Moscow, Radio and communications, 2002.

[14] Gribov M.M. Adjustable shock absorbers electronic Equipment Moscow, Soviet Radio, 1974.

[15] V.F.Borisov, O.P. Lavrenov, A.S. Nazarov, A.N. Chekmarev; Ed. A.S.Nazarov . Design of radio electronic facilities, Moscow, Science, 1996.

[16] V.P. Mayboroda. On the applicability of the influence functions determined from quasi-static experiments for the solution of dynamic viscoelasticity problems, Moscow, Polymer Mechanics, 1974, No. 3 , pp.537- 540 .

[17] V.V. Matveev, O.T. Bashta. On the influence of the shape of the cross-section and the type of rod deformation on the damping of their oscillations, Strength Problems, 1971, No. 10, pp.67-71,

[18] A.D. Mizhidon, M.B. Imayeov. Limit possibilities of the spatial 
vibroprotective system of a solid under impact perturbationsm Modern technologies. System analysis. Modeling, 2010, No. 1 (25) pp. 56-63

[19] M. Mirsaidov, T. Sultanov, Sh. O. Khudainazarov. "Own and established forced oscillations of a high-altitude structure with dynamic absorbers", Scientifican- technical magazine, Tashkent, J. Applied Mechanics, 2002, №-3, pp. 37-42.

[20] A.H. Naife. Perturbation method., Moscow, 1972

[21] Yu.M. Pleskachevsky, E.I. Starovoitov, D.V. Leonenko. "Mechanics of three-layered rods and plates connected with an elastic base", Moscow, Fizmatlit, 2011.

[22] Yu.N. Rabotnov." Elements of hereditary mechanics of solids". Moscow, Science, 1978.

[23] A.A.Samarskii, A.P. Mikhailov. "Math modeling". Ideas, Methods. Examples, Moscow, Fizmatlit, 2005.

[24] I.I. Safarov, "Oscillations and waves in dissipative - inhomogeneous media and structures". Tashkent, 1992.

[25] I.I. Safarov, M.Kh. Teshaev. "On the implementation of servo connections by the transferring body", Uzbek journal "Problems of mechanics", 2009, №3, pp.67-69

[26] E.I. Starovoitov, D.V. Leonenko. "Dynamics of round three-layer plates on an elastic base, with axisymmetric loaded", Problems of Strength, , 2010, №3, pp.113-124.

[27] E.I. Starovoitov, A.V. Yarovaya, D.V. Leonenko. “ Deformation of three-layer structural elements on an elastic foundation",Moscow, Fizmatlit, 2006.

[28] Yu.S. Urzhumtsev, V.P. Mayboroda. "Technical means and methods for determining the strength characteristics of structures from polymers, Moscow, Mechanical Engineering, 1984.

[29] K.V. Frolov, F.A. Furman. "Applied theory of outtake systems", Moscow, Mechanical Engineering, 1980.

[30] I.I.Safarov, M.Kh.Teshayev, M.Madjidov , "Natural Oscillations of Viscoelastic Lamellar Mechanical Systems with Poin Communications", Applied Mathematics, Vol. 5, pp.3018-3025, 2014

[31] I.I. Safarov, M.Kh.Teshaev, B.Z.Nuriddinov,O.Sh. Qilichov. Mathematical Modeling of Static Stress-Strain State of Parallel Tubes, Located in an Elastic Environment", Case Studies Journal, Volume 4 Issue 3, p.40-51, March-2015.

[32] Kolovsky M.Z." Automatic control of vibration protection systems", Moscow, Nauka, 1976

[33] Genkin M.D., Elezov V.G., Yablonsky V.V. "Methods of controlled vibration protection of machines", Moscow, Nauka, 1985.

[34] Safarov I.I., Axmedov M.Sh. "Free Oscillations of a Viscoelastic Toroidal Thin Shell with a Flowing Liquid", International Journal of Emerging Engineering Research and Technology, Vol. 6, Iss. 1, PP 114,2018

[35] Safarov I.I., Boltaev Z.I. "Propagation of Natural Waves on Plates of a Variable Cross Section", Open Access Library Journal, Volume 5 , E 4262, PP 1-29, 2018, https://doi.org/10.4236/oalib.1104262.

[36] Safarov I.I., Boltaev Z.I. "Methods for Assessing the Seismic Resistance of Subterranean Hydro Structures Under the Influence of Seismic Waves", American Journal of Physics and Applications. 6(2) PP 51-62. 2018. http://www.sciencepublishinggroup.com/j/ajpa.

[37] Safarov I. I., Kuldashov N.U., Boltaev Z.I. "Oscillations and Waves in a Layered Homogeneous Viscoelastic Medium”. International Journal of Emerging Engineering Research and Technology, Volume 6. Issue 3, PP 27-32, 2018.
[38] Safarov I.I, Teshaev M. H., Boltaev Z. I, Akhmedov M. Sh., "Mathematical modeling of dynamic processes in a toroidal and cylindrical shell interacting with a liquid". Raleigh, North Carolina, USA: Open Science Publishing, 2018.

[39] Safarov 1.1., Teshaev M.Kh., Boltaev Z. I. "Estimates of the dynamic Characteristics and stress-strain state of cylindrical and toroidal shells with a liquid under the action of dynamic loads". Raleigh, North Carolina, USA: Open Science Publishing, 2018.

[40] Safarov I.I., Marasulov A.M., Akhmedov M. Sh. "Impacts seismic waves on the viscoelastic cylindrical pipe with liquid", LAMBERT academic Publishing, 2018

[41] Safarov I.I., Boltaev Z.I., Teshaev.M.Kh. "Properties of Wave Motion in a Cylindrical Shell, Interacting with Viscous Liquid", Open Access Library Journal, Volume 5, pp.1-22, 2018

[42] Safarov I.I., Teshaev M.Kh., Boltaev Z.I . "Own Vibrations of Bodies Interacting with Unlimited Deformable Environment", Open Access Library Journal, Volume 5, e 4432, Access Library Journal, 5: PP 1 22, 2018, https://doi.org/10.4236/oalib.1104432

[43] Matsuzaki Y., Kobayashi S. "Nonlinear analysis of supersonic pane flatter of circular cylindrical shells”, Ibid., № 18, pp.103-111, 2018.

[44] Olson M.D., Fung Y.C." Supersonic flutter of circular shells subjected to internal pressure and axial compression ", Ibid.4, , 1966 , № 4, pp. 858-864

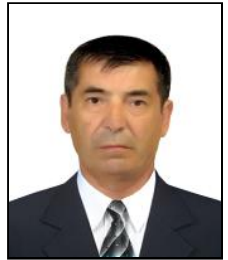

Safarov Ismoil Ibrokhimovich was born on Augus 10,1954 in the Bukhara region of the Republic of Uzbekistan. Doctor of Physics and Mathematics, Professor, in the field of mechanics of a deformable solid. In 1972-1977 he graduated from the Tashkent National University, in 1979-1983 and 1989-1992 he studied postgraduate and doctoral studies at the Moscow Institute of Electronic Engineering.

He works as the head of the department "Higher Mathematics" of the Tashkent Chemical Technology Institute. His scientific interests are the mechanics of solids and liquids.

1. Safarov II, Teshayev M.Kh. Vibrations of curvilinear tubes interacting with the medium. Analysis, concepts, methods. LAP, Lambert Academic Publishing (Germany). 2012. 245p.

2. Safarov II, Teshayev M.Kh. , Boltaev ZI Wave processes in mechanical waveguide. Fundamentals, concepts, methods. LAP, Lambert Academic Publishing (Germany). 2012, 275p.

3. Safarov I.I., Teshaev M. H., Madjidov M. Natural Oscillations of Viscoelastic Lamellar Mechanical Systems with Point Communications Applied Mathematics, 2014, 5, 3018-3025 (Springer USA).

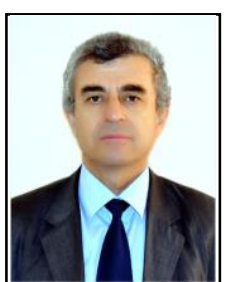

Teshaev Muhsin Khudoyberdieyvich, was born on February 5, 1961 in the Kagan district of the Bukhara region. In 1978, he entered Tashkent State University and graduated from the University in 1983 with degree in Mechanics. In 1993, in Almaty Nationa University he defended his thesis on the topic: "The dynamics of difficult mechanical systems with servo constraints". Author of more than 150 scientific articles. 